\title{
La contestation des projets d'infrastructures de transports : l'exemple du Grand Contournement Ouest (GCO) de Strasbourg
}

Opposition to transportation infrastructure projects: the case of the bypass road in the west of Strasbourg (Grand Contournement Ouest- GCO)

Lokalopposition gegen Verkehrsinfrastruktur: der Fall des Projekts von der Umgehungsstraße West der Region Straßburg (Grand Contournement Ouest GCO)

\section{Olivier Vergne}

\section{OpenEdition}

Journals

Édition électronique

URL : http://journals.openedition.org/rge/7227

ISSN : 2108-6478

Éditeur

Association des géographes de l'Est

Référence électronique

Olivier Vergne, « La contestation des projets d'infrastructures de transports : l'exemple du Grand

Contournement Ouest (GCO) de Strasbourg », Revue Géographique de l'Est [En ligne], vol. 57 / 3-4 | 2017, mis en ligne le 12 octobre 2018, consulté le 08 septembre 2020. URL : http://journals.openedition.org/ $\mathrm{rge} / 7227$

Ce document a été généré automatiquement le 8 septembre 2020

Tous droits réservés 


\section{La contestation des projets d'infrastructures de transports : l'exemple du Grand Contournement Ouest (GCO) de Strasbourg}

Opposition to transportation infrastructure projects: the case of the bypass road in the west of Strasbourg (Grand Contournement Ouest- GCO) Lokalopposition gegen Verkehrsinfrastruktur: der Fall des Projekts von der Umgehungsstraße West der Region Straßburg (Grand Contournement Ouest GCO)

\section{Olivier Vergne}

1 Le lundi 10 septembre 2018 vers $5 \mathrm{~h}$ du matin débutait l'évacuation de la ZAD (Zone A Défendre) de Kolbsheim où environ 200 opposants cherchaient à empêcher le début des travaux de déboisement devant permettre le lancement des travaux du Contournement Ouest de Strasbourg (COS), ou A 355, plus connue sous son ancien nom de Grand Contournement Ouest (GCO). En février 2018 c'était une autre ZAD, celle de Bure dans la Meuse qui était évacuée, un mois après l'annonce de l'abandon du projet d'aéroport de Notre-Dame-des-Landes. C'est d'ailleurs la contestation de ce projet qui avait donné naissance à la première « $\mathrm{ZAD}$ » apparue en 2007 dans le bocage nantais et qui a pu rassembler jusqu'à 500 personnes à certaines périodes (Subra, 2017). En octobre 2014, c'est une autre ZAD qui faisait la une de l'actualité, celle de Sivens dans le Tarn, où Rémi Fraisse, un jeune militant écologiste opposé au projet de barrage, avait trouvé la mort lors d'affrontements contre les forces de l'ordre. Malgré d'importantes différences de l'une à l'autre, toutes ces ZAD sont caractérisées par une occupation permanente des terrains concernés par un projet de nouvelle infrastructure, rendant de fait les travaux impossibles. Si la contestation de projets de nouvelles infrastructures n'est pas nouvelle ${ }^{1}$, le phénomène des ZAD marque une certaine radicalisation de cette contestation. 
2 Cette dimension conflictuelle liée aux projets d'aménagement ou d'équipement du territoire est, depuis les années 2000, l'objet d'une attention croissante des géographes (Cadoret, 2006 ; Laslaz, 2005 ; Lecourt, 2003 ; Mele et al., 2003 ; Subra, 2014). Si les projets d'infrastructure de transport ne sont pas les seuls concernés (que l'on pense au barrage de Sivens, au Center parc de Roybon, au centre d'enfouissement des déchets nucléaires de Bure, etc.), plusieurs projets liés au transport, tels que le projet d'aéroport de Notre-Dame-des-Landes (Renard J. et C. Rialland-Juin, 2013), celui de nouvelle voie ferrée à grande vitesse Lyon-Turin, ou de nouvelle autoroute de contournement de Strasbourg sont l'objet d'une vive contestation, qui peut conduire au ralentissement du projet, à sa modification, voire à son annulation. Cet article vise à analyser le cas du conflit autour du projet de Grand Contournement Ouest de Strasbourg, axe autoroutier envisagé depuis les années 1970, mais qui n'a toujours pas vu le jour, malgré un contexte de forte croissance de la métropole strasbourgeoise (Nonn, 2015 ; Woessner, 2009). Nous montrerons dans cet article pourquoi cet exemple est intéressant pour comprendre comment se structure une opposition locale à un projet de nouvelle infrastructure de transport, et plus largement comment tout projet de cette nature comporte une dimension politique, voire géopolitique au sens de rivalités de pouvoir sur le territoire ${ }^{2}$, qu'il convient d'expliciter pour une bonne compréhension de la dynamique du conflit, et des conditions de réalisation, ou d'abandon d'un projet de nouvelle infrastructure de transport.

\section{Les origines du projet de GCO, initialement consensuel}

\section{Résoudre le problème de la saturation de l'A35}

3 L'actuel projet de GCO doit permettre de résoudre un problème majeur concernant les déplacements dans la région strasbourgeoise : celui de la saturation, aux heures de pointe du matin et du soir, de la portion de l'A35 traversant l'agglomération de Strasbourg du nord au sud sur une vingtaine de kilomètres (axe à $2 \times 3$ voies). Avec en moyenne 160000 véhicules/jour, dont environ $10 \%$ de poids lourds, cet axe est un des plus fréquentés de France, avec un trafic qui continue d'augmenter. Dès 1973, le premier Schéma Directeur d'Aménagement et d'Urbanisme (SDAU) prévoyait d'ailleurs cette saturation et la nécessité de construire un autre axe plus à l'ouest pour compléter le réseau routier strasbourgeois. Cette situation est devenue depuis plusieurs années un sujet de mécontentement de plus en plus vif de la part des entreprises et des particuliers qui empruntent cet axe quotidiennement et qui subissent ainsi une perte de temps et un gaspillage de carburant importants. Ce problème est également préoccupant pour les 20 à $40 \%$ des habitants de l'agglomération vivant à proximité de l'autoroute et qui sont soumis à de fréquents dépassements des valeurs limites réglementaires de la qualité de l'air. Enfin, la saturation quotidienne de l'A35 menace l'attractivité économique de la métropole alsacienne dans la mesure où elle nuit à la compétitivité des entreprises locales et peut représenter un frein pour les investisseurs. Elle risque également de dégrader l'image de «ville verte " que s'est forgée Strasbourg depuis les années 1990 avec les efforts importants pour développer le réseau de tramway et l'usage du vélo.

4 Ce problème quotidien de saturation s'explique notamment par la situation particulière de l'A35, qui superpose quatre types de flux de natures différentes: les flux de transit 
longue distance transeuropéen nord-sud (accentué depuis l'instauration en 2005 d'une taxe poids lourds en Allemagne), les flux de transit régional (l'A35 est le seul axe nordsud en Alsace), les flux internes à l'agglomération (l'A35 permet de relier entre eux les grands axes urbains et est d'autant plus utilisée pour cela que le centre-ville est devenu très peu accessible en voiture avec la mise en place d'un tramway dans les années 1990), et les flux pendulaires entre l'agglomération et sa périphérie (pour lesquels l'automobile est, de loin, le moyen de transport le plus utilisé, malgré le développement depuis les années 1990 d'un réseau de cars interurbains par le Conseil départemental et d'un réseau de trains régionaux par le Conseil régional). L'A35 est donc à la fois un axe de transit et une " rocade ", celle-ci étant cependant incomplète dans la mesure où elle ne fait pas le tour de la ville, en raison de la frontière franco-allemande ${ }^{3}$.

5 Pour répondre à la saturation de l'A35 aux heures de pointe, plusieurs projets sont aujourd'hui portés, de façon plus ou moins active, par les trois grandes collectivités locales : modernisation de la rocade sud et projet d'accès au nord pour fluidifier l'accès au port, mise en place d'une ligne de bus interurbain en site propre entre Strasbourg et Wasselonne (TSPO pour Transport en site propre de l'ouest strasbourgeois), création d'une nouvelle voie routière nord-sud traversant les communes de la première couronne (VLIO pour Voie de liaison intercommunale ouest), requalification de l'A35 en boulevard urbain et réalisation du GCO.

\section{Croquis de localisation}

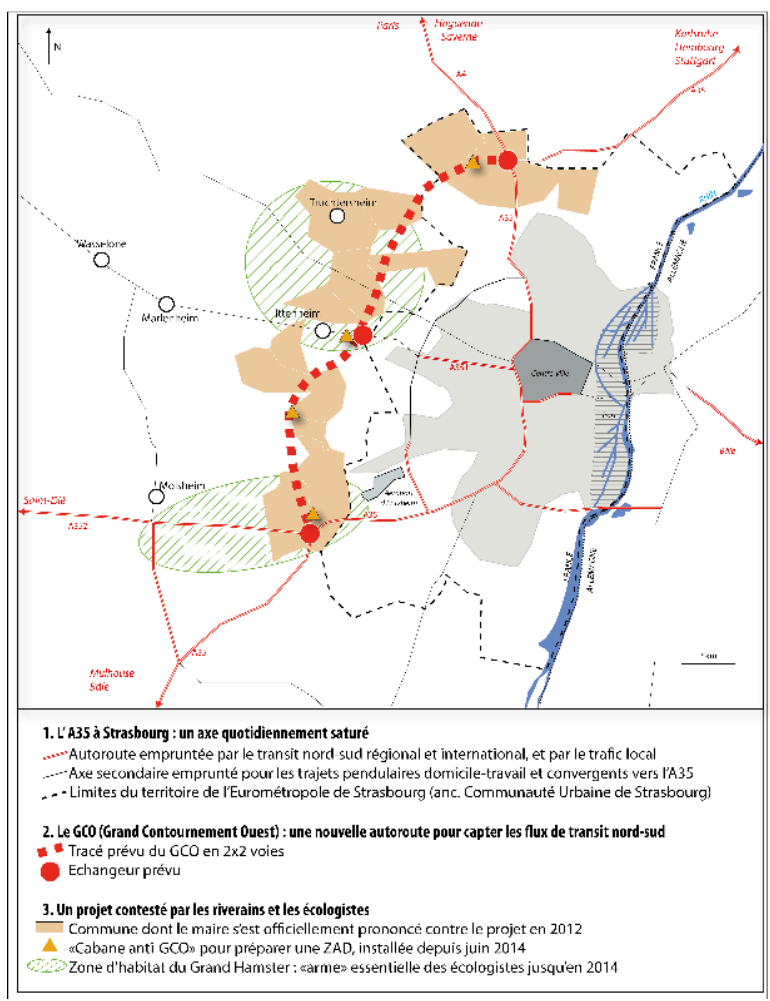

\section{Un projet initialement consensuel}

6 Avant de devenir un projet sur lequel vont s'opposer la gauche et la droite locale pendant les années 2000, le GCO apparaît comme relativement consensuel au sein de la classe politique locale au moment du «débat Bianco» de 1999. En effet, le Conseil 
Régional et le Conseil Général du Bas-Rhin, tous deux contrôlés par le centre-droit avec respectivement Adrien Zeller et Philippe Richert, soutiennent largement le projet, perçu comme bénéfique pour l'économie alsacienne. Du côté de la Ville de Strasbourg, la socialiste Catherine Trautmann, ayant dû quitter son fauteuil de maire pour celui de ministre de la Culture, annonce son soutien au projet dans un courrier envoyé au ministre des transports, Jean-Claude Gayssot (PC), et le maire par intérim Roland Ries (PS), soutient également le projet. Les élus écologistes acceptent également le projet, malgré la contestation de l'association Alsace Nature, et c'est d'ailleurs Dominique Voynet, alors ministre écologiste de l'aménagement, $\mathrm{du}$ territoire et de l'environnement, qui valide, avec Jean-Claude Gayssot, ministre des transports, le cahier des charges résultant du débat. Un tel consensus montre, qu'en ce début des années 2000, le paradigme d'un aménagement du territoire par de grands axes automobiles structurants incarnant la modernité, est toujours partagé par les acteurs politiques et techniques, dans la continuité par exemple, du "plan Vivien » approuvé en 1965. Ce premier plan directeur d'urbanisme pour l'agglomération strasbourgeoise prévoyait alors une série de pénétrantes et contournantes autoroutières, à une époque où la ville était encore conçue " comme un gigantesque système de distribution entre des flux automobiles d'origines et de destinations différentes » (Badariotti, 1996).

Dans les années 1990, les élus locaux et les services de l'Etat s'entendent alors sur les caractéristiques de l'axe qui aura pour vocation de capter le trafic de transit empruntant jusqu'alors l'A35, pour fluidifier la circulation sur cet axe et améliorer la liaison nord-sud européenne. Cependant, les élus alsaciens veulent éviter que le nouvel axe attire les poids lourds empruntant l'autoroute parallèle en Allemagne, ce qui justifie la décision de prévoir un péage sur le nouvel axe, une mesure qui présente également l'avantage de pouvoir accélérer la procédure, dans la mesure où un concessionnaire privé pourra réunir les fonds nécessaires beaucoup plus rapidement que l'Etat. Cette décision, qui ne fait pas débat à l'époque, constituera pourtant dans les années suivantes un des principaux arguments aux opposants au projet, et accréditera la représentation, largement partagée dans les rangs des opposants, selon laquelle le projet de GCO vise surtout à satisfaire les intérêts croisés des élus locaux et des acteurs privés du secteur du bâtiment et de la concession autoroutière.

\section{Un rythme d'avancement du projet soumis à la configuration politique locale}

\section{Années 2001-2008 : I'avancée rapide d'un projet porté par la droite locale aux commandes des trois grandes collectivités concernées}

8 Entre 2001 et 2008, le projet de GCO avance rapidement grâce à une configuration politique favorable. D'une part, les trois grandes collectivités concernées, à savoir le Conseil régional d'Alsace, le Conseil général du Bas-Rhin et la Mairie de Strasbourg4, sont toutes contrôlées par la droite. Si ces collectivités locales n'ont aucun pouvoir décisionnel quant à la construction d'une telle infrastructure (ce qui relève de la compétence du pouvoir central), l'unité qu'elles affichent en faveur du projet leur permet de porter une pression efficace sur les services de l'Etat, afin que ceux-ci s'engagent dans le projet. 
9 Ainsi, après que le projet ait été inscrit en 2003 sur la liste des «cinquante projets prioritaires à long terme » par le Comité Interministériel d'Aménagement du Territoire (CIADT), la Commission Nationale du Débat Public (CNDP) décide en février 2005 de ne pas organiser de débat public afin d'accélérer la procédure ${ }^{5}$, et ce malgré une opposition grandissante ${ }^{6}$. A l'échelon local, en 2006, dans le Schéma de Cohérence Territoriale de la Région Strasbourgeoise (SCOTERS), les communes de la région strasbourgeoise établissent leurs projets de développement territorial en prenant en compte la réalisation à court terme du GCO. En janvier 2008, le Premier ministre François Fillon signe la Déclaration d'Utilité Publique (DUP) en précisant le caractère « urgent » du projet. Le début des travaux est alors prévu en 2009 et la mise en service en 2012.

\section{8-2012: un projet retardé puis quasiment abandonné par le pouvoir socialiste.}

Dans ce contexte, les socialistes strasbourgeois vont faire du GCO un enjeu pour les élections municipales de 2008, grâce auxquelles ils espèrent reconquérir la mairie perdue en 2001. Dans cette perspective, l'opposition au GCO, aux côtés des agriculteurs, des riverains et des écologistes, va apparaître comme un combat pouvant être électoralement porteur, et ce pour trois raisons. Premièrement, cette position permet de capter les suffrages des nombreux électeurs strasbourgeois qui semblent sensibles aux arguments des opposants qui ont alors déjà organisé plusieurs manifestations à Strasbourg. Deuxièmement, elle permet de s'opposer clairement à la droite et de critiquer l'action menée en faveur du projet. Troisièmement, elle permet de préparer une alliance avec les Verts strasbourgeois, en vue d'une fusion des listes PS et Verts au $2^{\mathrm{e}}$ tour des municipales.

11 En 2008, en partie grâce à cette stratégie, les socialistes alliés aux écologistes parviennent à reprendre à la droite la Mairie de Strasbourg ${ }^{7}$. Cette nouvelle configuration politique met alors fin au « front commun » des trois collectivités locales en faveur du projet. Ainsi, en décembre 2008, R. Ries (maire PS de Strasbourg) et J. Bigot (président PS de la Communauté Urbaine de Strasbourg) écrivent au Président de la République Nicolas Sarkozy, pour lui demander d'abandonner le projet de GCO. La procédure va alors fortement ralentir, et ce malgré la présence au gouvernement de Philippe Richert, l'un des plus ardents défenseurs du GCO, président (UMP) de la Région Alsace et ministre des collectivités locales.

12 Pour la droite locale qui continue de soutenir le projet, l'objectif est alors de faire signer les derniers décrets permettant le début des travaux avant les élections présidentielles de 2012, car celles-ci pourraient consacrer une victoire socialiste, ce qui risquerait de conduire à un abandon du GCO conformément aux souhaits des socialistes strasbourgeois. C'est pourquoi une tentative d'accélération est engagée en février 2012 par Philippe Richert (UMP) à la tête du Conseil régional, et Guy-Dominique Kennel (UMP), à la tête du Conseil général du Bas-Rhin, qui font voter par leurs assemblées respectives une subvention de 3,25 millions d'euros chacune à Vinci, le concessionnaire pressenti ${ }^{8}$. Malgré cette accélération de la droite locale, le décret définitif d'attribution du marché à Vinci ne sera pas signé avant l'élection de F. Hollande. Dès juin 2012, le nouveau gouvernement socialiste retire le projet à Vinci au motif que le groupe avait 
dépassé le délai pour clore son tour de table financier, ce qui est perçu à Strasbourg, comme un abandon du projet par l'Etat.

\section{Depuis 2013 : un projet relancé après la campagne de la $\mathrm{CCl}$ et le revirement des socialistes strasbourgeois.}

13 A la suite de ce quasi-abandon, le projet va cependant être relancé à l'initiative de la Chambre de Commerce et d'Industrie (CCI) de Strasbourg et du Bas-Rhin, qui parvient à convaincre les socialistes strasbourgeois de la nécessité du GCO. Pour cela, la CCI déploie une stratégie en plusieurs bandes :

14 Premièrement, elle rappelle l'attachement du patronat local au projet, en invitant ses membres à signer une pétition en ligne à l'adresse du ministre des transports, et elle commande également un sondage à l'entreprise TNS Sofres, auprès de 1000 dirigeants d'entreprises locaux, dont $92 \%$ se disent favorables à une relance du GCO.

Deuxièmement, elle organise une importante campagne de sensibilisation à l'attention du monde économique, et également du grand public, grâce à une brochure de 44 pages intitulée " GCO tous gagnants », très bien documentée et d'une grande qualité graphique, expliquant pourquoi le GCO est indispensable pour l'agglomération strasbourgeoise. A cette brochure s'ajoutent également un blog dédié (www.gcotousgagnants.com), et plusieurs annonces en pleine page dans les $\mathrm{DNA}^{9}$, ou sur les panneaux d'affichage urbain.

16 Troisièmement, le projet est présenté comme indispensable à la région strasbourgeoise, non seulement pour des raisons économiques, mais également pour des raisons de santé publique et de qualité de l'air. Ainsi, le vocabulaire utilisé dans la brochure n'hésite pas à filer la métaphore médicale en rappelant que, sans le GCO, la région strasbourgeoise est "en voie de thrombose", ou d' "asphyxie ", au sens propre comme au figuré. En élargissant ainsi l'enjeu du GCO à la question sensible de la qualité de l'air à Strasbourg ${ }^{10}$, la CCI peut espérer rallier à sa cause de nombreux strasbourgeois.

17 Enfin quatrièmement, pour permettre aux socialistes strasbourgeois d'accepter la relance du projet, sans renier leur opposition au GCO depuis les années 2000, la CCI propose un projet modifié. Ainsi, le dimensionnement de l'axe est revu à la baisse (2x2 voies au lieu de $2 \times 3$ voies), et il est présenté comme étant indissociable d'une requalification de l'A35 en boulevard urbain, avec feux, vitesse limitée à $70 \mathrm{~km} / \mathrm{h}$, voies dédiées au transport en commun et au covoiturage.

18 La manœuvre est un succès pour la CCI, car à la suite de cette campagne, les socialistes strasbourgeois vont passer du camp des opposants à celui des partisans au GCO, permettant la relance du projet.

19 Ainsi, en novembre 2012, le ministre des transports Frédéric Cuvillier commande, à la demande de la CCI et de Roland Ries et Jacques Bigot (respectivement maire PS de Strasbourg et président PS de la Communauté Urbaine de Strasbourg), une nouvelle expertise auprès du Conseil Général de l'Environnement et du Développement Durable (CGEDD). Le rapport, rendu en novembre 2013, préconise alors une relance du projet mais en intégrant les modifications proposées par la CCI. Dans ces conditions, Roland Ries se déclare favorable à ce GCO remanié, et écrit à nouveau au ministre des transports, mais pour lui demander, cette fois-ci, non plus d'abandonner le projet, mais 


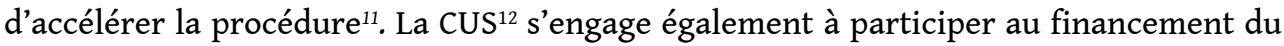
projet avec la Région et le Conseil général à raison de trois tiers identiques, en précisant que les fonds fournis par la CUS seront exclusivement consacrés au réaménagement de l'A35, qui traverse le territoire de la CUS (contrairement au GCO qui contourne la CUS par l'ouest). En février 2014, un nouvel appel public à concurrence est lancé pour la concession du GCO.

Ce ralliement des socialistes strasbourgeois au projet, vécu comme une traîtrise de la part des opposants, et notamment par leurs alliés écologistes à la Mairie, s'explique par plusieurs raisons.

\section{L'enjeu politique}

21 Premièrement, contrairement à 2008, les socialistes peuvent estimer que, pour les municipales de 2014, il est plus intéressant électoralement de soutenir le GCO que de s'y opposer. En effet, l'accent mis par les promoteurs du projet, notamment la CCI, sur l'amélioration de la qualité de l'air à Strasbourg que permettrait la réalisation du GCO, en limitant l'engorgement sur l'A35 traversant l'agglomération, a pu convaincre un certain nombre d'électeurs strasbourgeois. Le coût politique d'une telle stratégie pour les socialistes pourrait être la remise en cause de l'alliance avec les écologistes, pouvant conduite à la perte de la Mairie en $2014^{13}$, mais ces derniers, s'ils dénoncent ce changement de position des socialistes, renouvellent en 2014 leur alliance de 2008 au second tour des municipales. Cette alliance est en effet nécessaire pour eux s'ils souhaitent conserver un certain nombre de sièges au conseil municipal et communautaire ${ }^{14}$. Les mauvais résultats du PS à l'échelle de la communauté urbaine vont alors placer dans un premier temps les écologistes dans une position de force au sein du conseil communautaire : le nombre d'élus de la majorité PS-EELV n'y dépasse alors que d'une voix celui de l'opposition et le PS devra donc absolument avoir le soutien des écologistes pour obtenir une majorité lors des votes. Une telle configuration est alors trop dangereuse pour le PS, en désaccord avec les écologistes sur de nombreux dossiers, notamment celui du GCO. Robert Hermann (le futur président PS de la communauté urbaine) décide alors de s'allier avec la droite pour gérer la communauté urbaine, devenue Eurométropole. Dans ce contexte, les écologistes perdent en influence par rapport à la précédente mandature, ce qui affaiblit grandement le camp des opposants au GCO.

\section{L'enjeu économique}

Deuxièmement, les enjeux économiques liés au projet expliquent également le revirement des socialistes strasbourgeois : le lancement de ce grand chantier, et ensuite l'entretien de l'infrastructure, est en effet un levier important pour dynamiser le secteur local du BTP et maintenir des emplois, dans un contexte de faible croissance et de réduction des dépenses publiques ${ }^{15}$. En mars 2015, les entreprises du secteur organisent d'ailleurs une grande manifestation à Strasbourg pour réclamer de nouveaux investissements aux responsables politiques locaux, et pour demander, entre autres moyens de relancer l'activité, le début rapide des travaux du GCO. Malgré les accusations de "soumission au lobby du BTP et de la CCI » ${ }^{16}$ qui leur sont portées par les anti-GCO, R. Ries et R. Hermann assument tout à fait cet argument économique dans un courrier qu'ils envoient au ministre des transports en août 2014 pour lui demander 
d'accélérer le calendrier, afin de compenser la baisse annoncée des investissements de leurs collectivités en raison de leurs fortes contraintes budgétaires.

\section{L'enjeu de l'aménagement urbain et de l'attractivité de Strasbourg}

Troisièmement, le nouveau projet, avec requalification de l'A35, passant d'une autoroute $2 \times 3$ voies en boulevard urbain, permet d'envisager des aménagements urbains en bordure de l'axe. En effet, les nuisances sonores devenant beaucoup moins importantes, plusieurs hectares pourront constituer des réserves foncières intéressantes pour de futurs projets urbains au service de l'attractivité d'une ville qui ambitionne de devenir une grande métropole rhénane et européenne. C'est notamment le cas des terrains situés à l'arrière de la gare où un pôle tertiaire a plusieurs fois été évoqué depuis les années 2000.

La nouvelle version du GCO est également présentée par la Mairie socialiste comme une condition pour améliorer la qualité de l'air dans l'agglomération, et une opportunité de développer les transports en commun sur l'A35 requalifiée en boulevard urbain (avec des voies réservées pour le covoiturage et les bus urbains). Si ces enjeux se posent pour toutes les grandes villes françaises, ils prennent une importance particulière à Strasbourg, qui a misé une partie de son attractivité sur son image de «ville durable » avec le " premier réseau cyclable de France ", et le " premier réseau de tramway de France $»^{17}$.

Malgré ce qui apparaît donc, à partir de 2013, comme un consensus politique local pour le GCO, une incertitude quant à sa réalisation continue de planer jusqu'au début des travaux en septembre 2017. En effet, une opposition importante s'est constituée au fil des années, faisant intervenir plusieurs catégories d'acteurs, aux modes d'actions et aux intérêts divergents.

Schéma des acteurs intervenant sur le projet de GCO

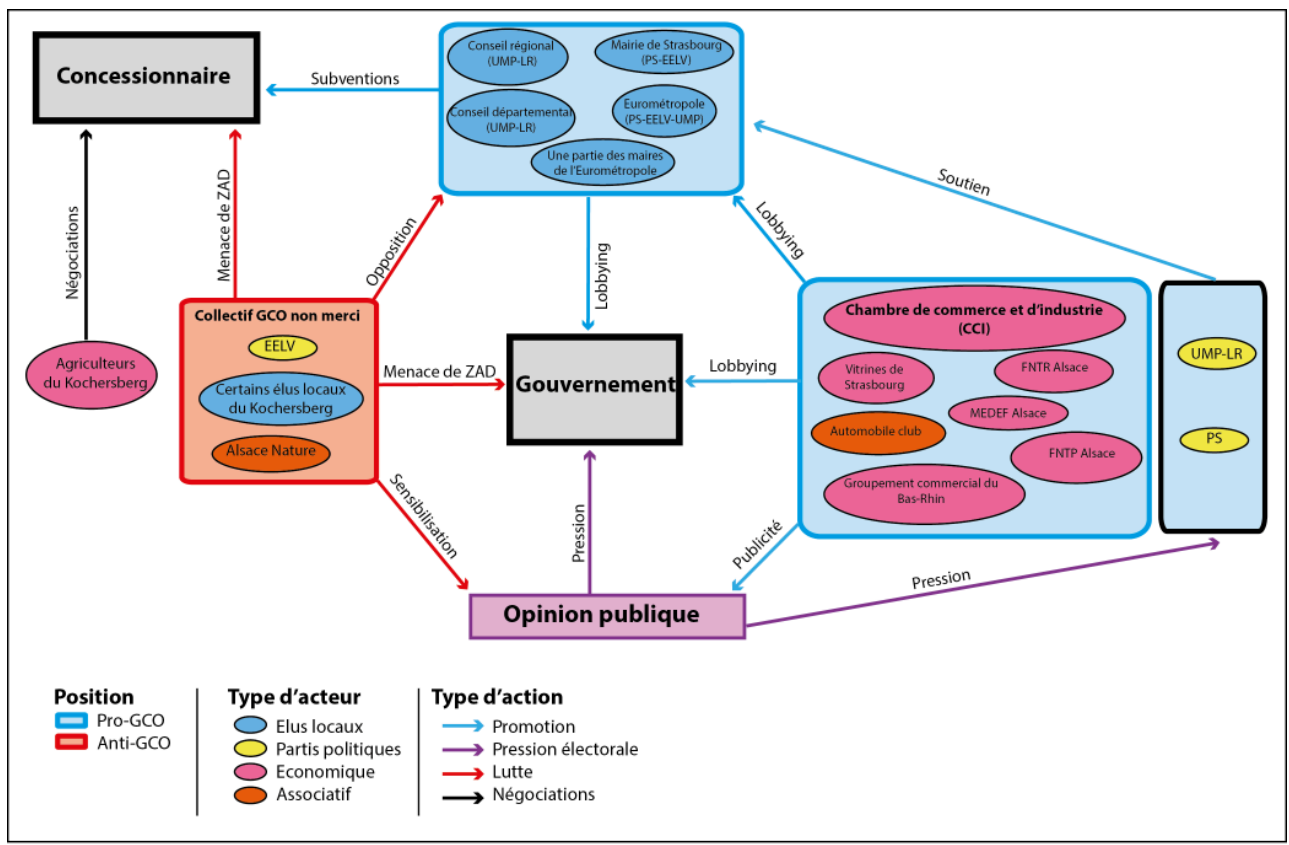


Chronologie du projet et configuration politique locale

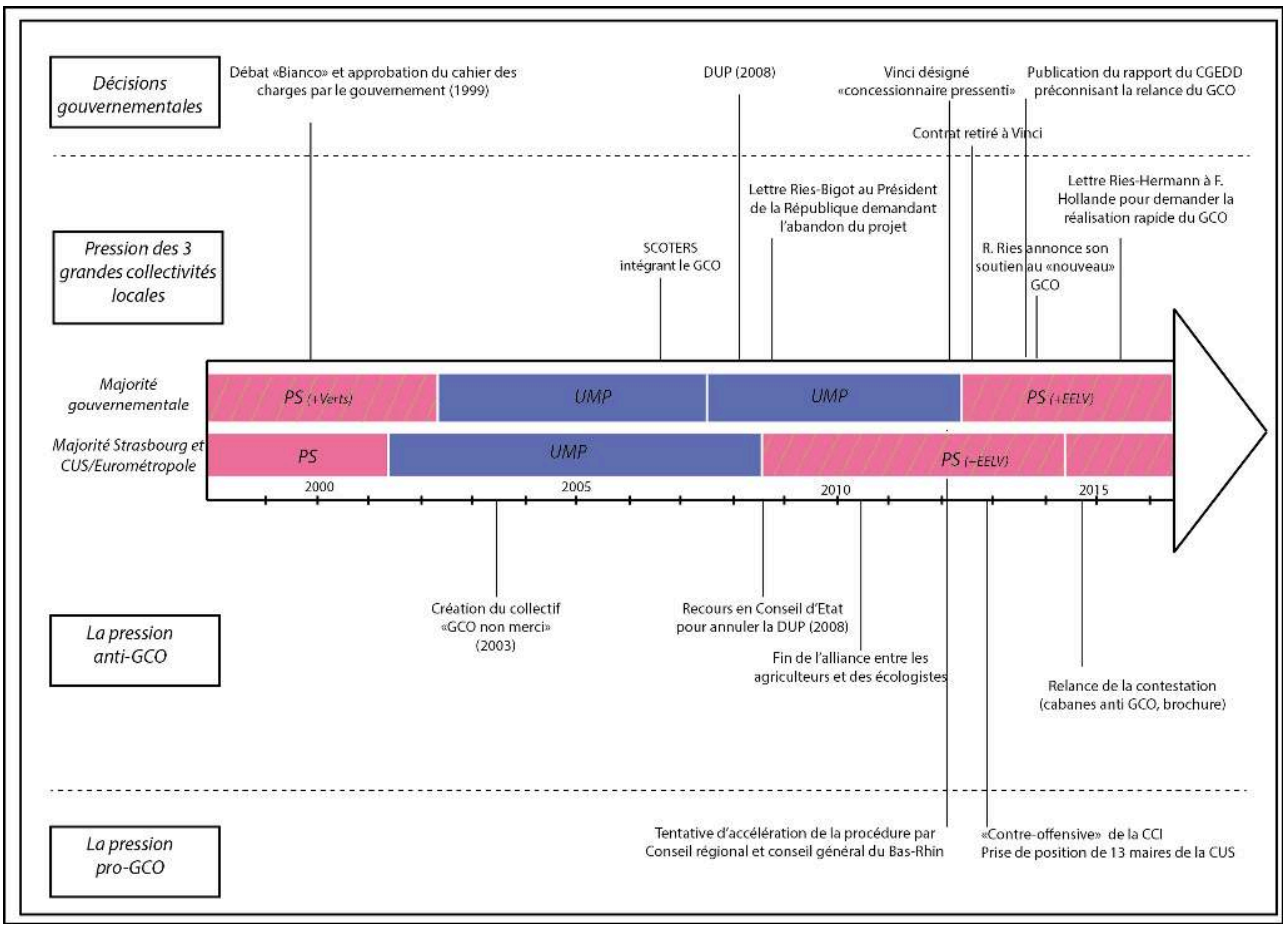

Publicité pour la réalisation du GCO publiée en pleine page des Dernières Nouvelles d'Alsace (DNA) par la CCI à l'été 2012.

Source : gco2016tousgagnants.com, blog de la CCI Strasbourg/Bas-Rhin consacré à la campagne en faveur du GCO.

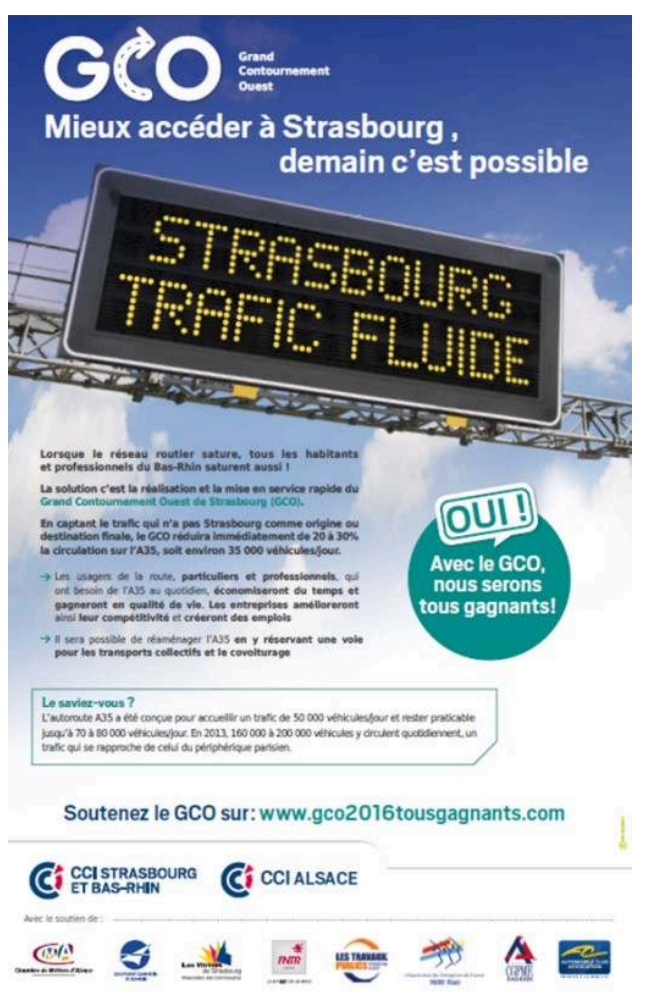




\section{Une opposition au GCO persistante et multiforme}

\section{Le collectif GCO non-merci : l'alliance fragile des agriculteurs, des écologistes et des riverains.} des opposants au projet pour en faire un thème de campagne en vue des élections municipales de 2008. Ce mouvement d'opposition au GCO s'est structuré et a acquis une certaine puissance grâce à l'alliance, au sein du Collectif GCO non-merci créé en 2003, de trois types d'acteurs: les riverains, représentés par des élus locaux et certaines associations ${ }^{18}$, qui craignent les nuisances et une perte de valeur de leur patrimoine immobilier ; les agriculteurs, représentés notamment par la FNSEA locale, qui rejettent l'artificialisation de 300 ha de terres agricoles à très haut rendement, ainsi qu'une perte de revenus estimée à $10 \%$ pour 200 exploitants; et les écologistes, représentés par le parti Les Verts (puis EELV) et la fédération Alsace Nature, qui redoutent une hausse de la circulation automobile ${ }^{19}$ et de la pollution atmosphérique dans la région, ainsi qu'une atteinte à la biodiversité, mettant notamment en danger la survie d'une espèce protégée emblématique, le Grand Hamster d'Alsace (cricetus cricetus). tenter de faire annuler le projet: commande d'une contre-expertise à un cabinet indépendant pour mettre en évidence les failles du projet ${ }^{20}$, action en justice par un recours au Conseil d'Etat ${ }^{21}$, organisation de manifestations dans le Kochersberg et à Strasbourg, comme celle organisée en 2006 regroupant 2000 personnes et une centaine de tracteurs, bloquant la circulation dans la ville pendant plusieurs heures. Cette opposition semble alors parvenir à convaincre une large part de l'opinion publique locale, notamment strasbourgeoise, comme le montre un sondage réalisé en 2008 par les Dernières nouvelles d'Alsace, le grand quotidien régional, qui montre que « $59 \%$ des lecteurs sont contre le $\mathrm{GCO} »^{22}$.

Durant ces années 2000, le collectif GCO non-merci apparait alors assez puissant, d'autant plus qu'il est soutenu par les socialistes strasbourgeois. Cependant, cette puissance repose largement sur une alliance fragile car quasiment " contre-nature » : celle entre les militants écologistes représentés par Alsace Nature et le parti EELV, et les agriculteurs, notamment représentés par la FNSEA, un syndicat agricole largement critiqué par les écologistes pour sa pratique intensive de l'agriculture.

Durant les années 2000, cette alliance fragile entre écologistes et agriculteurs ne sera pas remise en cause, car le Collectif GCO non-merci apparaîtra davantage aux agriculteurs comme un mouvement de riverains porté par quelques élus locaux que comme un mouvement d'écologistes, même si c'est l'association Alsace Nature qui a été à l'initiative de sa création. Ainsi, jusqu'en 2010, le porte-parole du collectif est ainsi Daniel Zeter, vice-président du conseil général et maire UDF-UMP de Stutzheim, une des communes traversées par le projet, et d'autres élus locaux comme Justin Vogel, vice-président UMP du conseil régional et président de la communauté de communes $\mathrm{du}$ Kochersberg, sont des figures importantes de l'opposition au projet. Cette configuration évolue en 2010 quand le nouveau porte-parole du collectif, Luc Huber, élu de la commune de Pfettisheim, se présente comme candidat EELV aux élections régionales (comme il le fera également aux élections cantonales de 2011 et législatives de 2012). Les agriculteurs décident alors de mettre fin à leur participation aux actions 
du collectif, qui «est devenu écolo $»^{23}$, et ils continuent leur opposition au projet de façon indépendante.

Pour les agriculteurs, l'opposition au GCO ne répond pas aux mêmes objectifs que ceux des écologistes et des riverains. Pour ces deux derniers, le GCO est inacceptable à aucune condition, contrairement aux agriculteurs, qui sont prêts à accepter le nouvel axe si les compensations financières sont suffisamment élevées. Ainsi, à partir de 2013, une majorité des agriculteurs (ceux membres de la FNSEA et des Jeunes agriculteurs) va même cesser les actions contre le projet. Jusqu'en 2012, ils continuaient à s'y opposer indépendamment du collectif GCO non merci on l'a vu, car ils estimaient que les mesures financières compensatoires prévues par Vinci étaient insuffisantes. A partir de la relance du projet en 2013, des négociations sont menées auprès des concessionnaires candidats par la Chambre d'agriculture du Bas-Rhin et par la FNSEA, avec le soutien de plusieurs maires et d'élus cantonaux, pour obtenir de la part des concessionnaires le maximum de compensations financières possibles, outre celles prévues par la loi. Les agriculteurs entendent profiter de leur position de force: si le concessionnaire doit légalement réaliser certaines mesures de compensation environnementales ${ }^{24}$, il n'a aucun moyen coercitif envers les agriculteurs pour que ceux-ci aménagent leurs terrains en ce sens. Or, comme il n'existe que très peu d'hectares non cultivés dans cette partie de l'Alsace, les mesures de compensation environnementales devront donc nécessairement être réalisées sur des terres déjà cultivées. Pour que leur dossier de candidature soit crédible et pour que le délai de réalisation de l'autoroute, et donc le coût du chantier soit le plus réduit possible, chaque concessionnaire doit donc satisfaire les agriculteurs. Ainsi, alors que la législation prévoit un remembrement de 6 600 ha pour réorganiser l'ensemble du parcellaire, les agriculteurs demandent au concessionnaire le financement nécessaire au remembrement de $10000 \mathrm{ha}^{25}$. Pour les agriculteurs, cette attitude est légitime car ils s'estiment être les premiers directement impactés économiquement par le projet, beaucoup plus que les écologistes qui se battraient eux pour de vagues idées, et qui peuvent se permettre de rester dans une logique d'opposition frontale car ils n'ont, eux, rien à perdre.

31 Afin de renforcer leur position dans leurs négociations avec le concessionnaire, les agriculteurs de la FDSEA vont participer périodiquement aux actions du collectif GCO non-merci comme lors de la grande manifestation à Strasbourg en octobre 2016. Cependant l'année suivante, les mêmes agriculteurs organiseront leur propre manifestation pour dénoncer les mesures environnementales que Vinci a dû ajouter à son projet suite à un avis du CNPN (Conseil National pour la Protection de la Nature) et à la pression d'Alsace Nature. En fin de manifestation les agriculteurs déverseront du fumier et des pneus devant le siège de l'association à Strasbourg. 
Manifestation des agriculteurs contre le GCO à Stutzheim-Offenheim en mars 2012, pour demander à Vinci, concessionnaire pressenti à l'époque, de revoir à la hausse les indemnités financières des mesures compensatoires.

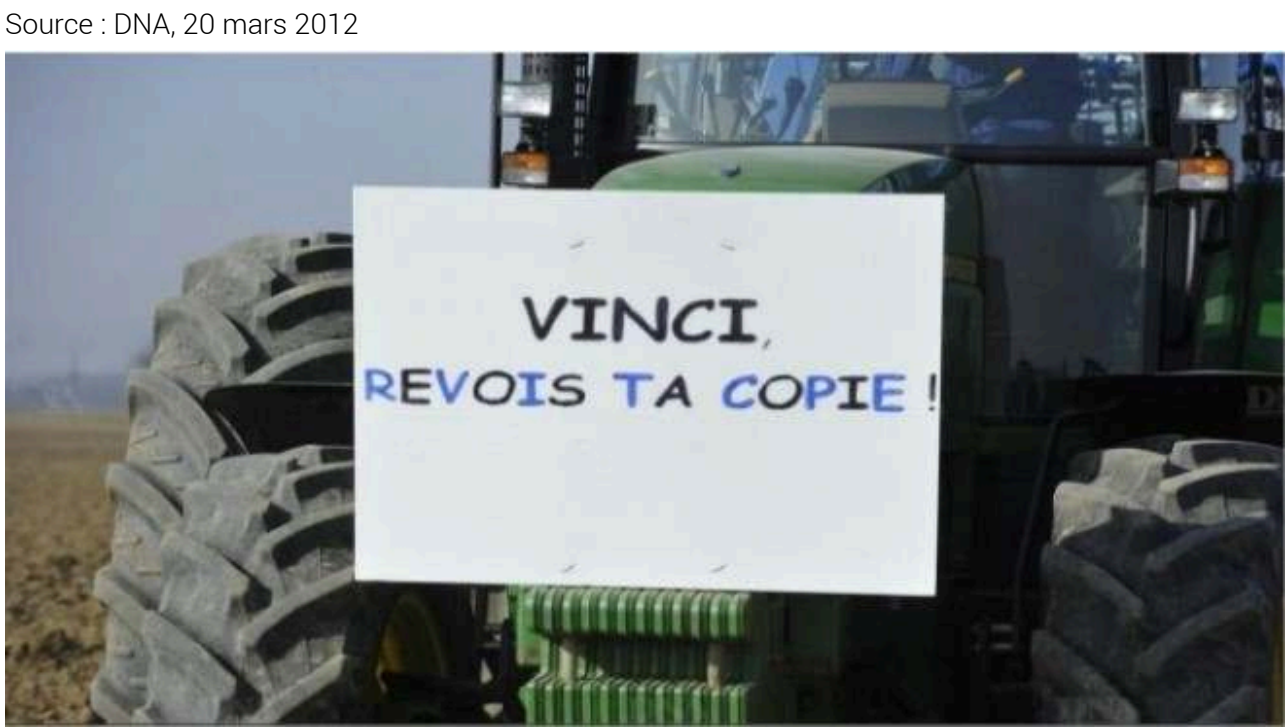

\section{Les tensions autour du Grand Hamster d'Alsace, révélateur des divergences entre écologistes et agriculteurs}

Un autre point de tension entre les agriculteurs et les écologistes résident dans le dossier du Grand Hamster d'Alsace, une espèce protégée rare en France, seulement présente dans la région strasbourgeoise ${ }^{26}$.

Dans la région du Kochersberg traversée par le futur GCO, les écologistes dénoncent depuis plusieurs décennies la maïsiculture intensive qui a provoqué une importante érosion de la biodiversité et qui a notamment mené à la quasi-disparition du Grand Hamster, espèce considérée comme nuisible jusque dans les années 1970, mais qui est, " grâce " au projet de GCO, devenue emblématique de la lutte pour la biodiversité dans la région strasbourgeoise, qui abrite les derniers habitats alsaciens d'une espèce autrefois très présente dans la région.

Jusqu'en 2012, le Grand Hamster a été l'une des «armes" les plus efficaces des écologistes, car l'animal était protégé par la Convention de Berne ${ }^{27}$ et par la directive « Habitats » de 1992. A la suite d'une pétition déposée en 2010 auprès du Parlement européen, Alsace Nature avait réussi, en juin 2011, à faire condamner la France par la Cour de justice de l'Union européenne pour le non-respect de la directive « Habitats » en raison des mesures insuffisantes prises pour la préservation du Grand Hamster. Cette action auprès de l'UE a probablement contribué aux difficultés de Vinci à réunir les fonds à temps en 2012, car la Banque européenne d'investissement avait refusé de participer à un projet mettant en cause la politique de protection du grand hamster menée par la Commission.

Cependant, cette protection du Grand Hamster par les écologistes est très mal acceptée par les agriculteurs locaux et par de nombreux riverains et élus locaux. Pour les agriculteurs, les mesures de protection du Grand Hamster sont perçues comme une menace pour leur activité agricole et comme une critique directe vis-à-vis de leurs pratiques intensives. Pour de nombreux élus des communes autour de Strasbourg, cette 
protection du Grand Hamster est également perçue comme un frein au développement économique et à l'accueil de nouvelles activités et infrastructures. Ainsi, en septembre 2012, Guy-Dominique Kennel (UMP), président du conseil général du Bas-Rhin ${ }^{28}$, avait contesté la décision du gouvernement socialiste de protéger 9300 ha de terres en périphérie strasbourgeoise autour des terriers de Grand Hamster ${ }^{29}$, pour répondre à la condamnation européenne de 2011 et éviter de nouvelles amendes. En novembre 2012, c'était au tour d'une cinquantaine de communes de la grande périphérie strasbourgeoise de déposer un recours au Conseil d'Etat contre cet arrêté, considéré comme un cadeau du gouvernement socialiste aux «bobos» strasbourgeois, représentés par la majorité PS-EELV, accusés de vouloir mettre "sous cloche » la campagne autour de la ville.

A partir de 2013, "l'arme " Grand Hamster va cependant devenir beaucoup moins efficace pour les écologistes : en décembre 2013, le comité permanent de la Convention de Berne décide de lever la procédure de surveillance des mesures prises par la France pour la sauvegarde du Grand Hamster, au motif que les mesures nécessaires avaient été prises (alors même que le projet de GCO venait d'être relancé un mois plus tôt). En avril 2014, la Commission des pétitions du Parlement européen décide de clore le dossier d'Alsace Nature, qui avait, par une nouvelle pétition, demandé la condamnation de la France pour son projet de GCO. Pour les partisans du GCO, l'argument selon lequel la réalisation de la nouvelle autoroute conduirait à la disparition du Grand Hamster est infondée car des mesures importantes de protection ont été prévues. Ainsi, en mai 2014, le Conseil régional d'Alsace lance un programme de protection, baptisé Alister (pour "Alsace Life Hamster»), entrant dans le cadre des programmes Life soutenus financièrement par la Commission européenne, pour un coût estimé à 3 millions d'euros, avec le soutien de la Chambre d'agriculture. Cette opération permet aux proGCO de montrer que le Grand Hamster n'est pas condamné par la nouvelle autoroute, mais peut au contraire y trouver les conditions de sa protection grâce aux ambitieuses mesures de compensation environnementales prévues. L'objectif est ici de diffuser l'idée selon laquelle le GCO est une chance et non une menace pour le Grand Hamster.

Dessin illustrant l'opposition au GCO, et faisant référence à la célèbre photographie de la Place Tian'Anmen de 1989.

Les écologistes, représentés par le Grand hamster, sont assimilés aux manifestants se battant pour la démocratie, face aux chars du régime, qui prennent ici la forme des engins de chantier. Les sentiments des écologistes sont ici bien explicités : I'opposition au GCO est un combat asymétrique pour une juste cause face à un système politico-économique tout puissant, coupable d'un déni de démocratie et d'injustice, ce qui légitime l'organisation d'une ZAD. Source : site internet d'une association locale pour la protection du grand hamster d'Alsace (hamster-alsace.fr, consulté en septembre 2015) 


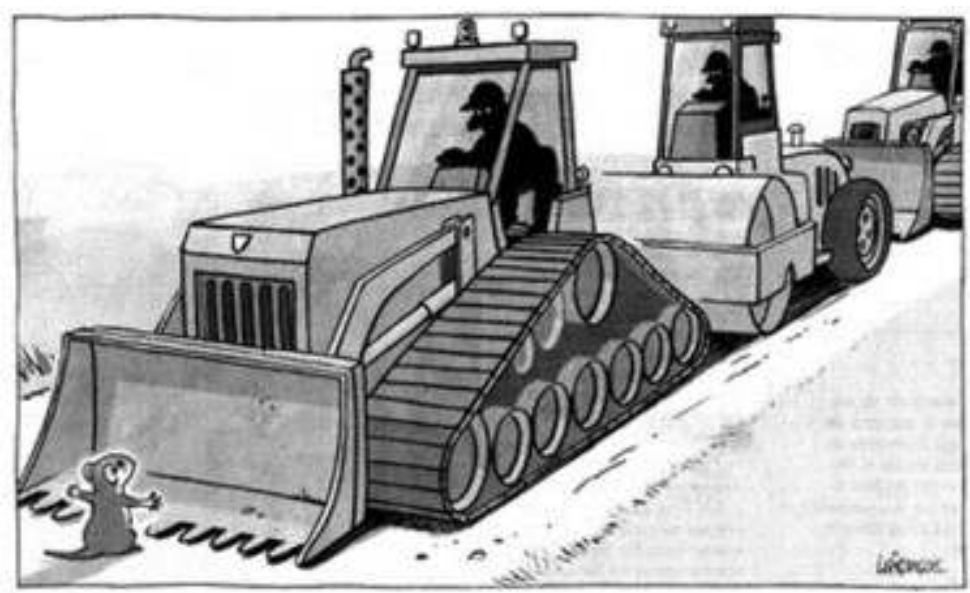

\section{Vers une radicalisation de l'opposition avec la constitution d'une ZAD?}

37 Au cours des années 2010, le Collectif GCO merci perd donc, on l'a vu, plusieurs soutiens importants : élus socialistes strasbourgeois, agriculteurs, instances européennes. Face à cet affaiblissement et à l'épuisement des recours administratifs, le collectif, qui regroupe essentiellement à partir de 2013 des écologistes et des riverains, menace l'Etat et le concessionnaire de créer une Zone A Défendre (ZAD) pour empêcher physiquement le chantier, sur le modèle des médiatiques ZAD de Notre-Dame-des-Landes ou de Sivens. Ce glissement vers un mode de contestation radicale s'explique par l'épuisement de tous les recours légaux, et par le sentiment d'une injustice et d'un déni de démocratie, provoqué par ce qui est perçu comme une soumission des élus (notamment des socialistes strasbourgeois) aux lobbies du BTP et de la CCI, face auxquels les militants écologistes ne peuvent faire le poids, et malgré des expertises indépendantes concluant à l'inefficacité du GCO à réduire les bouchons de l'A35.

Ainsi, le rapport TTK de 2005 qui avait conclu à l'inefficacité du GCO n'a jamais été considéré par les promoteurs du projet. De plus, en 2006, le dossier d'enquête publique prévoyait un report de trafic sur le GCO de seulement $5 \%$ et la Commission d'enquête publique avait conclu, dans son rapport de 2006, que le désengorgement n'était «ni l'enjeu ni l'objectif du GCO $»^{30}$.

L'objectif de cette menace de ZAD retardant le chantier est de dissuader le concessionnaire à s'engager dans le projet, qui serait alors perçu comme trop risqué en termes de coûts et de délais. Le collectif a alors en tête la date de janvier 2018 qui marquera la fin de la validité de la DUP de 2008 et il faut donc empêcher que les travaux commencent avant cette date. Dans «l'attente » du début des travaux, le collectif GCO non merci prépare cette ZAD en construisant, à partir de juin 2014, plusieurs « cabanes anti-GCO » sur le tracé prévu. Le rôle de ces cabanes est alors triple : il permet, lors des inaugurations de créer des évènements médiatiques rappelant l'opposition au GCO grâce à la présence notamment des élus EELV de Strasbourg. D'autre part, les cabanes, posées en bord de route sont des supports de communication pour rappeler aux automobilistes l'opposition au GCO, pour signifier qu'il ne résoudra pas le problème des bouchons et qu'il sera payant. Enfin, ces cabanes permettent de symboliser l'occupation future du terrain. En constituant des «bases » pour les futurs zadistes, elles symbolisent la menace de la ZAD. 
Cette menace laisse alors planer une certaine incertitude sur la réalisation du GCO, mais on peut se poser la question de l'efficacité d'une telle démarche dans cette riche région agricole de l'ouest de Strasbourg, marquée par l'importance du vote conservateur et par une certaine méfiance à l'égard des mouvements radicaux et alternatifs. Certes, les idées écologistes peuvent y connaître un certain succès, comme en Alsace en général, mais, comme le montre l'attitude des élus écologistes strasbourgeois qui siègent avec les socialistes malgré les nombreuses divergences, l'écologie politique est plutôt ici dans une démarche de négociation, et non d'opposition frontale, conformément à la culture politique alsacienne. Le recrutement des zadistes ne pourra donc pas se faire localement et c'est pourquoi le collectif cherche à développer les liens avec d'autres mouvements zadistes et à inscrire le GCO sur la «liste de grands projets inutiles", ce qui pourrait pousser certains zadistes extérieurs à la région à venir occuper le terrain. Cependant, le risque est là d'attirer des éléments radicaux et violents qui desserviraient le mouvement, particulièrement dans cette région largement conservatrice et marquée par une forte présence économique et sociologiques des agriculteurs, dont une majorité assimile les zadistes à des casseurs. A ces difficultés tenant au contexte socio-politique local, s'ajoutent également des difficultés d'ordre davantage physique, en raison de la nature linéaire du projet, qui rend l'occupation du chantier plus difficile que dans le cas d'une infrastructure ponctuelle, et en raison du paysage de champs ouverts qui rend l'occupation illégale du terrain plus compliquée que dans le cas d'un paysage fermé de bocage comme dans la région nantaise où s'est installée la ZAD contre le projet d'aéroport de Notre-Dame-desLandes (Subra, 2017). A partir de l'été 2017, une ZAD parvient cependant à prendre forme dans une zone boisée de la commune de Kolbsheim, avec le soutien du maire et de plusieurs élus locaux (par exemple la député LREM Martine Wonner) et nationaux (par exemple l'eurodéputé José Bové). Cette mobilisation parvient à faire retarder de plusieurs mois les travaux de déboisement du bois de Kolbsheim, préalable aux travaux. Les mois suivants seront marqués par plusieurs avis négatifs au sujet des mesures compensatoires prévues par Vinci, mais sans remettre en cause le projet : en août 2017, le Centre National pour la Protection de la Nature (CNPN) rend un premier avis négatif en des termes très sévères ce qui pousse les services de l'Etat (DREAL) à exiger une amélioration du dossier de la part de Vinci, malgré le caractère uniquement consultatif de cet avis. En janvier 2018, le CNPN rend un 2e avis négatif, ce qui n'empêche cependant pas le Gouvernement de prolonger la DUP, arrivée à échéance, jusqu'en 2026. En février 2018, c'est l'Autorité Environnementale, émanant du Conseil Général pour l'Environnement et le Développement Durable, qui rend également un avis négatif, et en juin 2018, à l'issue de l'enquête publique, la commission d'enquête publique émet également un avis défavorable vis-à-vis des mesures environnementales compensatoires prévues par le concessionnaire. Malgré ces avis négatifs, le préfet de la région Grand Est signe fin août 2018 les arrêtés autorisant le début des travaux, et la ZAD de Kolbsheim, regroupant une quinzaine d'occupants permanents, est évacuée début septembre 2018, malgré la présence de 200 opposants, ce qui permet de lancer les travaux.

«Cabane anti-GCO » installée en juin 2015 sur la commune d'Ittenheim, au bord de la RN4.

Photographié par l'auteur, septembre 2015 


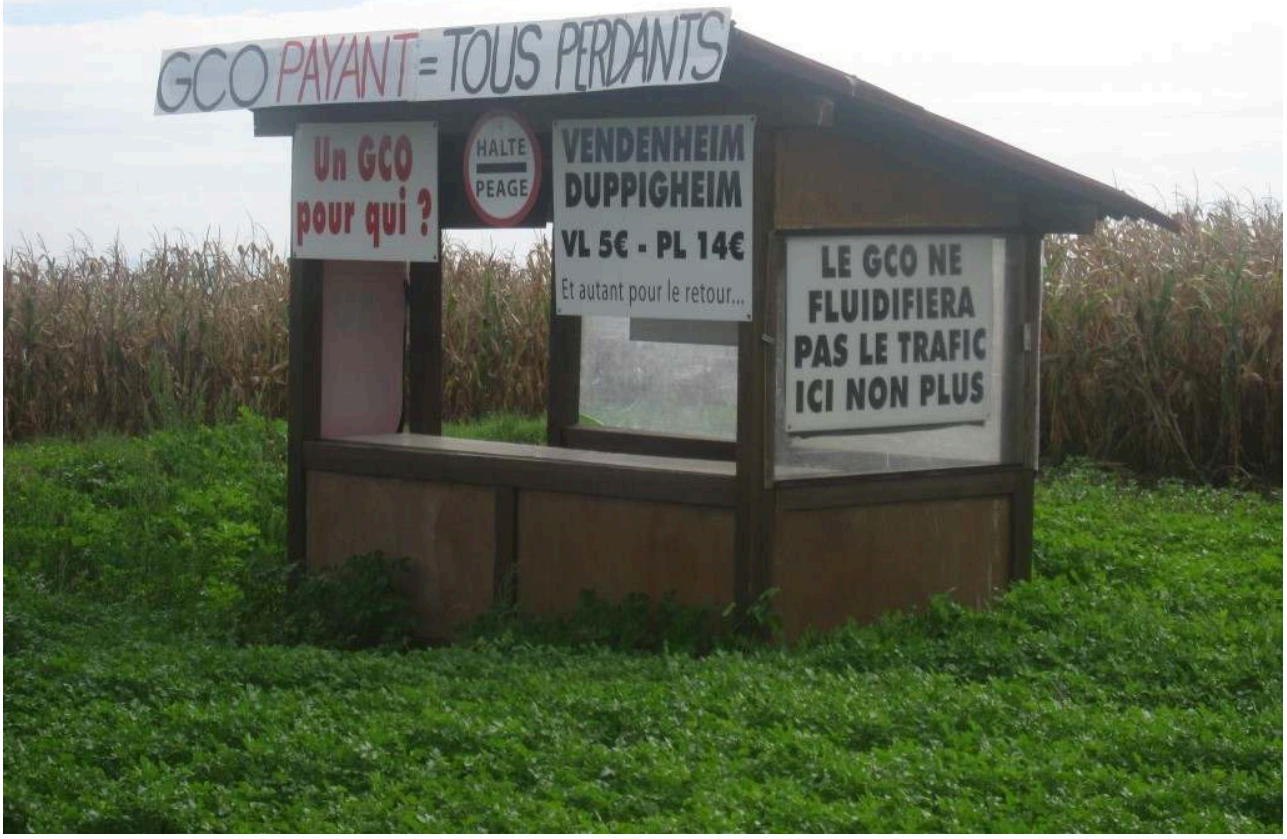

Paysage ouvert du Kochersberg où est prévu le GCO (au fond la cathédrale de Strasbourg)

Photographié par l'auteur, septembre 2015

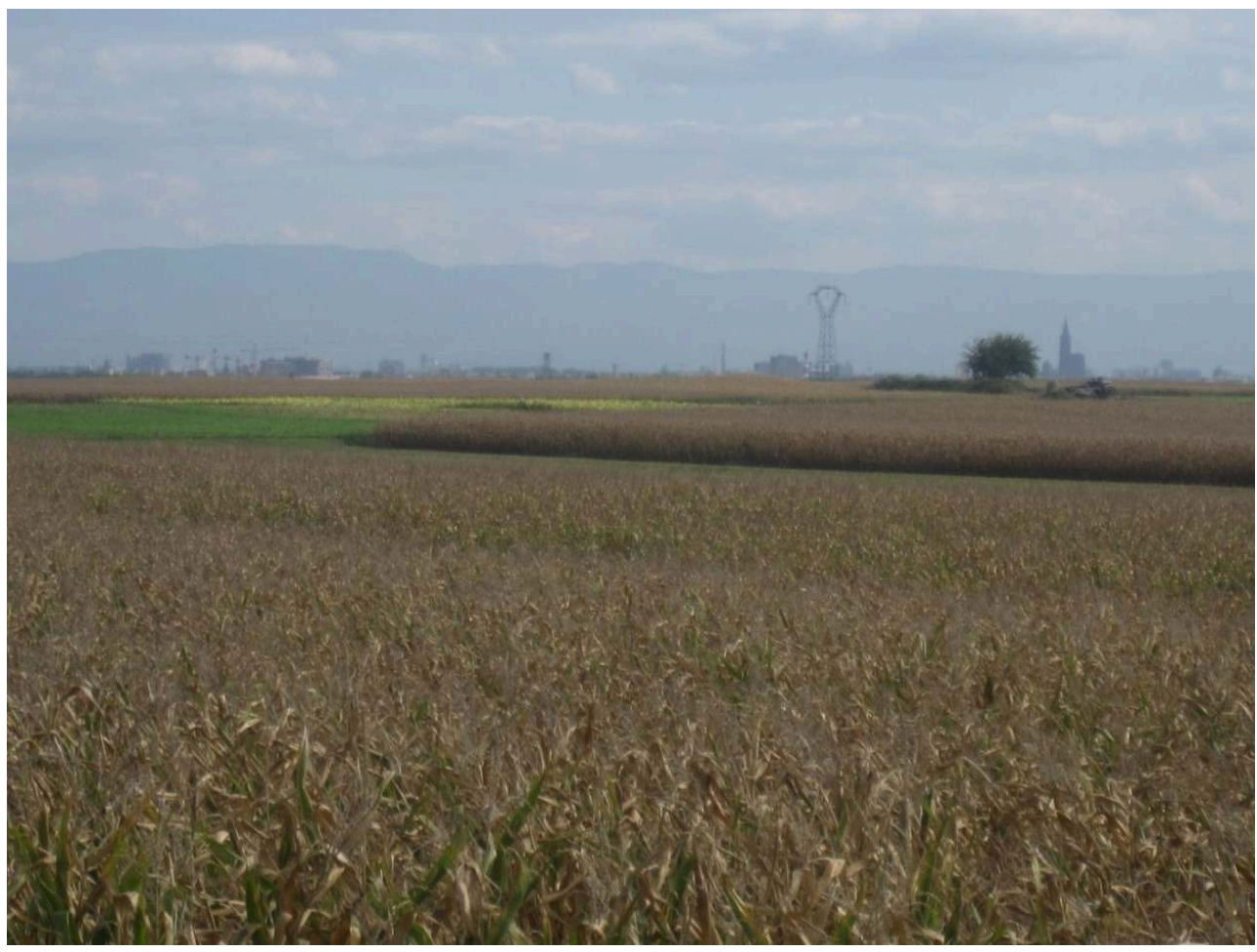




\section{Conclusion} mettre en évidence le rôle déterminant des élus locaux, donc de la configuration politique locale, dans le rythme d'avancement d'un tel projet de nouvelle grande infrastructure de transport, quand bien même ces élus locaux n'ont aucun pouvoir décisionnel en la matière. En effet, dans un contexte de restrictions budgétaires et de compétitions entre territoires pour attirer les investissements de l'Etat, celui-ci s'engagera prioritairement sur les projets soutenus par un "front commun» des collectivités concernées, comme c'est le cas pour le GCO depuis 2013, de façon transpartisane.

Or, comme le montre l'attitude des socialistes strasbourgeois dans ce dossier, la position des élus locaux peut être largement soumise aux enjeux électoraux locaux et donc à l' "opinion publique » locale. Pour les opposants, comme pour les partisans du projet, la capacité à influencer cette opinion publique locale constitue l'un des enjeux essentiels, car elle déterminera en grande partie la position des acteurs politiques. Les discours, les décisions et les actions des acteurs politiques et des responsables des collectivités locales concernées, résultent ainsi de la pression d'autres acteurs (associations de riverains, associations écologistes, agriculteurs, CCI, etc.), lesquels jouent un rôle majeur dans l'avancée, le retardement, voire l'abandon d'une nouvelle infrastructure de transport.

Plus largement, cet exemple montre bien que l'aménagement du territoire, après avoir connu son "âge d'or" dans les Trente Glorieuses marquées par le paradigme moderniste et par l'absence de remise en question de l'action publique, est aujourd'hui devenu un enjeu conflictuel. Autour de ces projets contestés se cristallisent des représentations contradictoires de ce qu'est l'intérêt général entre différents groupes sociaux, ce qui pose la question de la légitimité des décisions des pouvoirs publics en ce domaine.

Ces conflits d'aménagement sont ainsi, à bien des égards, des révélateurs de la crise politique actuelle en France et le développement du phénomène de ZAD montre l'échec des tentatives de concertation mises en œuvre depuis les années 1990 qui étaient censées apaiser les conflits relatifs aux projets d'aménagement du territoire (Subra, 2014).

\section{Références}

Badariotti D., 1996, « Projet politique et urbanisme : le Strasbourg de Pierre Pflimlin (1959-1983) », Espace géographique, tome 25, n¹, pp. 53-64.

Cadoret A., 2011, « Analyse des processus conflictuels. Le cas du littoral du LanguedocRoussillon », L’Espace géographique, 3/2011 (Tome 40), p. 231-244.

Laslaz L., 2005, Les zones centrales des Parcs Nationaux alpins français (Vanoise, Ecrins, Mercantour) : des conflits au consensus social ? Contribution critique à l'analyse des processus territoriaux d'admission des espaces protégés et des rapports entre sociétés et politiques d'aménagement en milieux montagnards, thèse de doctorat en géographie, Université de Savoie, $644 \mathrm{p}$

Lecourt A., 2003, Les conflits d'aménagement : analyse théorique et pratique à partir du cas breton, thèse de doctorat en géographie et aménagement, Université de Rennes 2 Haute-Bretagne, 363 p. 
Mele P., Larrue C. \& Rosemberg M., coord., 2003, Conflits et territoires, P.U. François Rabelais, Tours, coll. perspectives «Villes et territoires », 224 p.

Nonn H., 2015, L'Alsace actuelle, Strasbourg, Presses Universitaires de Strasbourg, 204 p.

Renard J. et C. Rialland-Juin, 2013, « Le projet d'aéroport de Notre-Dame-des-Landes : les rebonds d'un aménagement conflictuel », geoconfluences.ens-lyon.fr (4/2/2013)

Subra P., 2014, Géopolitique de l'aménagement du territoire, Paris, Armand Colin, $2^{\mathrm{e}}$ édition, 350p.

Subra P., 2017, « De Notre-Dame-des-Landes à Bure, la folle décennie des « zones à défendre » (2008-2017)», Hérodote 2017/2 (N 165), p. 11-30.

Woessner R., 2009, L'Alsace, territoire(s) en mouvement, Strasbourg, Do Bentzinger éditeur, 184p.

Documents émanant des acteurs du conflit :

CCI de Strasbourg et du Bas-Rhin, 2012, GCO 2016: Tous gagnants, Strasbourg, 44p.

Conseil Général de l'Environnement et du Développement Durable, 2013, Expertise sur les

déplacements dans la périphérie de l'agglomération strasbourgeoise, MEDDE, Paris, $120 \mathrm{p}$

Collectif GCO non-merci, 2015, Dix solutions pour faire sauter les bouchons, Strasbourg, 24p

CUS - Service mobilité, 2013, Nouvelle donne, nouvelle mobilité - Nouvelle A35 et boulevard Nord-Sud, Strasbourg, $102 \mathrm{p}$

Rapport de la Commission d'enquête publique, 2006, 299p

\section{NOTES}

1. Contestation contre le camp militaire du Larzac dans les années 1970, contre la centrale nucléaire de Plogoff à la même période, contre le TGV Méditerranée dans les années 1990, etc.

2. Pour reprendre la définition d'Yves Lacoste (LACOSTE Y., 1993 Dictionnaire de la géographie, de la géopolitique aux paysages, Paris, Armand Colin, 224p.)

3. Il n'existe, au niveau de Strasbourg, qu'un seul franchissement autoroutier sur le Rhin, au sud de l'agglomération, par le pont Pierre Pflimlin, ouvert en 2002, mais qui débouche sur un axe en $2 \times 1$ voies côté allemand.

4. Le Conseil régional d'Alsace et le Conseil général du Bas-Rhin ont toujours été contrôlés par la droite, contrairement à la Mairie de Strasbourg, tenue par le PS depuis l'élection de Catherine Trautmann en 1989, mais reconquise par la droite en 2001 derrière Fabienne Keller.

5. Pour argumenter ce choix, la CNDP s'appuie sur la décision du CIADT de 2003, et considère que la concertation avait déjà eu lieu de manière approfondie depuis 1999 entre les collectivités, les organismes économiques, les associations et le public, ce qui a conduit à prévoir de nombreuses mesures compensatoires pour limiter les nuisances et les impacts environnementaux.

6. Voir II.

7. Au $1^{\text {er }}$ tour, la liste de R. Ries (PS) recueillera $44 \%$, et l'ensemble des listes de gauche ne recueillera que $47 \%$, dont $6 \%$ pour les écologistes menés par Alain Jund. Au second tour, l'alliance entre le PS et les Verts remporte le $2^{\text {nd }}$ tour avec $58 \%$ face à la maire sortante F. Keller (UMP) 
8. Tentative d'accélération qui provoque un recours du groupe écologiste et socialiste au Conseil Régional, dénonçant la précipitation et l'opacité dans laquelle sont prises ces décisions.

9. Les Dernières Nouvelles d'Alsace (DNA) est le principal quotidien strasbourgeois et bas-rhinois. 10. La CCI rappelle ainsi dans sa brochure que, selon le plan de protection de l'atmosphère de l'agglomération de Strasbourg (PPA), 80000 habitants sont quotidiennement concernés par des niveaux de pollution aux particules fines dépassant les seuils critiques. Pour la CCI, la réalisation du GCO permettrait de réduire largement ce chiffre.

11. Dans un communiqué co-rédigé avec $P$. Richert, président du Conseil régional, et G-D. Kennel, président du Conseil général, R. Ries et J. Bigot écrivent en effet qu'ils souhaitent «qu'aucun retard ne soit pris dans la reprise de la procédure de mise en concession de l'ouvrage autoroutier [...], mais également dans l'engagement des études puis de travaux pour le réaménagement de l'A35 actuelle », DNA, $12 / 12 / 2013$

12. Communauté urbaine de Strasbourg, devenu le $1^{\mathrm{er}}$ janvier 2015 l'Eurométropole de Strasbourg.

13. Dans un contexte national difficile pour la gauche, de nombreuses villes socialistes passeront à droite lors de ces municipales, et la liste fusionnée au $2^{\text {nd }}$ tour Ries (PS)-Jung (EELV) ne l'emportera que de justesse à Strasbourg avec $47 \%$ des voix, deux points seulement devant la liste UMP-Modem-UDI menée par F. Keller.

14. A. Jund, tête de liste EELV au $1^{\mathrm{er}}$ tour obtiendra le poste de vice-président de l'Eurométropole en charge de l'urbanisme.

15. Le concessionnaire devrait investir entre 500 et 750 millions euros, et favoriser la création de 1500 emplois pendant les 2 ans du chantier, puis 50 emplois permanents pour l'entretien.

16. Brochure " 10 solutions pour faire sauter les bouchons ", GCO non merci. D'autant que l'un de vice-présidents de la CCI est Georges Lingenheld, PDG de Lingenheld, important groupe alsacien de BTP, qui figure parmi les 4 concessionnaires en lice pour le marché.

17. Site internet de l'Eurométropole de Strasbourg strasbourg.eu

18. Par exemple l'Association de la Défense pour la Qualité de Vie de Duttlenheim.

19. Dans une brochure publiée en janvier 2015, intitulée « 10 solutions pour faire sauter les bouchons", le collectif GCO non merci explique que la réalisation d'un nouvel axe nord-sud sera inefficace à régler un problème essentiellement dû à des trajets pendulaires, et que le GCO ne permettra pas de "faire sauter les bouchons ».

20. Rapport TTK, Karlsruhe, 2005 «A35 - Grand contournement ouest de Strasbourg- Expertise du dossier et comparaison de scénarios comparatifs contrastés "

21. Recours déposé en mars 2008 par Alsace Nature, douze communes, deux communautés de communes regroupant 25 communes, trois sections cantonales de la FDSEA, la SCI du Château de Kolbsheim.

22. DNA, 18 février 2008

23. Entretien avec un agriculteur, juin 2015.

24. Selon le cahier des charges, le concessionnaire doit prévoir 500 ha de terres pour reconstituer un habitat pour le Grand hamster, ainsi que 200 ha pour recréer des zones humides en compensation de celles de la vallée de la Bruche qui sera traversée par le GCO.

25. En 2015, le total des compensations demandé par les agriculteurs dans leurs revendications est de 30 millions d'euros. (Entretien Chambre d'agriculture du Bas-Rhin, juin 2015)

26. Appelé aussi «hamster d'Europe " (cricetus cricetus), il est surtout présent dans une bande allant de l'Europe orientale à l'Asie centrale, ainsi que dans quelques régions d'Europe occidentale (Alsace, Pologne, etc.)

27. Etablie en 1979 sous l'égide du Conseil de l'Europe, et signée par l'Union Européenne.

28. Qui, compte tenu de la géographie électorale bas-rhinoise représente les élus de droite nonstrasbourgeois majoritaires au Conseil Général face aux élus de gauche minoritaires, élus de Strasbourg 
29. L'arrêté du 6 août 2012 du ministère de l'environnement prévoit que toute construction dans les zones protégées autour des terriers recensés (600 mètres autour de chaque terrier) doit faire l'objet d'une demande de dérogation, autorisée en cas de mesures de compensation (les promoteurs d'un projet d'aménagement doivent trouver - et payer - des agriculteurs prêts à cultiver du blé ou de la luzerne, afin de reconstituer un habitat propice au hamster), ce qui est perçu comme un "gel des terres" par des nombreux élus des territoires concernés par ce périmètre de protection.

30. Rapport de la commission d'enquête publique, p147

\section{RÉSUMÉS}

Depuis le début des années 2000, les grands projets d'aménagement du territoire sont de plus en plus difficiles à mettre en œuvre en raison du rejet qu'ils suscitent dans une partie de l'opinion. En cette fin des années 2010, plusieurs projets en France sont ainsi combattus au moyen de Zones A Défendre (ZAD) sur le modèle de celle de Notre-Dame-des-Landes. C'est par exemple le cas du projet de Grand Contournement Ouest (GCO) dans la région strasbourgeoise, envisagé dans les années 1970, mais toujours non réalisé à ce jour en raison d'une forte opposition locale qui cherche aujourd'hui à constituer une ZAD. Cet article propose de montrer comment se structure une opposition locale à un grand projet d'aménagement du territoire, comment un tel projet entre en jeu dans les rivalités politiques locales, et quels acteurs il fait intervenir, avec leurs intérêts, leurs stratégies et leurs représentations propres.

Since the beginnings of 2000 decade, the implementation of large scale land planning projets is increasingly difficult, because of public opposition. In the end of the 2010's, several projects are fought in France, by the strategy of Zone A Défendre (ZAD), that could be translated by Place to protect, following the model of Notre-Dame-des-Landes, against a new airport near the city of Nantes. Among these ZAD is the one against the projet of Grand Contournement Ouest (GCO), near the city of Strasbourg. This project had been planned during the 1970's but is still not realised because of a local opposition that try to constitute a new ZAD. This article aims to explain how is built a local fight against a large-scale land planning project, how such a project can be a key issue in local politics fights, and who are the actors concerned, with their own aims, their strategies and their vision of the future of their territory.

Seit dem Anfang der 2000er die großen Raumordnungsprojekte werden wegen der Ablehnung, die sie in einem Teil der öffentlichen Meinung erwecken, immer schwieriger durchzuführen. Im heutigen Ende der 2010er mehrere Großprojekte werden in Frankreich mittels « Zonen zu verteidigen» (auf Französisch: Zone A Défendre, abk. ZAD) bekämpft, auf dem Modell von der « ZAD » von Notre-Dame-des-Landes. Es ist zum Beispiel der Fall des Projekts von der Umgehungsstraße West der Region Straßburg (auf Französisch: Grand Contournement Ouest, abk. GCO), das in den 1970er betrachtet wurde und bleibt heutzutage immer noch nicht erreicht, aufgrund einer starken Lokalopposition, die heute versucht, eine « ZAD » zu gründen. Dieser Artikel schlägt vor, $\mathrm{zu}$ zeigen, wie sich eine Lokalopposition gegen ein großes Raumordnungsprojekt sich strukturiert, wie ein solches Projekt zwischen den lokalen politischen Rivalitäten ins Spiel kommt, und welche Beteiligter involviert werden, mit ihren Interessen, ihren Strategien und ihren eigenen Darstellungen. 
INDEX

Mots-clés : aménagement du territoire, transports, conflit d'acteurs, luttes écologistes, géopolitique locale

Schlüsselwörter : Raumordnungsprojekte, Verkehr, Konflikte zwischen Akteuren, Umweltkämpfe, lokal Geopolitik

Keywords : land planning, transports, conflicts between actors, ecological struggles, local geopolitics

\section{AUTEUR}

\section{OLIVIER VERGNE}

<olivier.vergne@univ-rennes2.fr> PRAG Université Rennes 2, Place du Recteur Henri Le Moal, 35000 Rennes, Doctorant au Centre de Recherches et d'Analyses Géopolitiques (CRAG) - Université Paris 8, 2 rue de la Liberté, 93526 Saint-Denis 